\title{
Epigenetically Regulated Chromosome 14q32 miRNA Cluster Induces Metastasis and Predicts Poor Prognosis in Lung Adenocarcinoma Patients
}

\author{
Margarita González-Vallinas ${ }^{1,2}$, Manuel Rodríguez-Paredes ${ }^{3}$, Marco Albrecht ${ }^{4,5}$, \\ Carsten Sticht ${ }^{6}$, Damian Stichel ${ }^{4,7}$, Julian Gutekunst ${ }^{3}$, Adriana Pitea ${ }^{8}$, \\ Steffen Sass ${ }^{8}$, Francisco J. Sánchez-Rivera ${ }^{9}$, Justo Lorenzo-Bermejo ${ }^{10}$, \\ Jennifer Schmitt ${ }^{1}$, Carolina De La Torre ${ }^{6}$, Arne Warth ${ }^{1,11}$, Fabian J. Theis ${ }^{8}$, \\ Nikola S. Müller ${ }^{8}$, Norbert Gretz ${ }^{6}$, Thomas Muley ${ }^{11,12}$, Michael Meister ${ }^{11,12}$, \\ Darjus F. Tschaharganeh,13, Peter Schirmacher ${ }^{1}$, Franziska Matthäus ${ }^{4,14}$, \\ and Kai Breuhahn ${ }^{1}$
}

\begin{abstract}
Most lung cancer deaths are related to metastases, which indicates the necessity of detecting and inhibiting tumor cell dissemination. Here, we aimed to identify miRNAs involved in metastasis of lung adenocarcinoma as prognostic biomarkers and therapeutic targets. To that end, lymph node metastasisassociated miRNAs were identified in The Cancer Genome Atlas lung adenocarcinoma patient cohort (sequencing data; $n=449$ ) and subsequently validated by qRT-PCR in an independent clinical cohort $(n=108)$. Overexpression of miRNAs located on chromosome $14 \mathrm{q} 32$ was associated with metastasis in lung adenocarcinoma patients. Importantly, Kaplan-Meier analysis and log-rank test revealed that higher expression levels of individual 14q32 miRNAs (mir-539, mir-323b, and mir487a) associated with worse disease-free survival of never-smoker patients. Epigenetic analysis including DNA methylation microarray data and bisulfite sequencing validation demonstrated that the induction of $14 \mathrm{q} 32$ cluster correlated with genomic
\end{abstract}

hypomethylation of the $14 \mathrm{q} 32$ locus. CRISPR activation technology, applied for the first time to functionally study the increase of clustered miRNA levels in a coordinated manner, showed that simultaneous overexpression of $14 \mathrm{q} 32$ miRNAs promoted tumor cell migratory and invasive properties. Analysis of individual miRNAs by mimic transfection further illustrated that miR-323b-3p, miR-487a-3p, and miR-539-5p significantly contributed to the invasive phenotype through the indirect regulation of different target genes. In conclusion, overexpression of $14 \mathrm{q} 32$ miRNAs, associated with the respective genomic hypomethylation, promotes metastasis and correlates with poor patient prognosis in lung adenocarcinoma.

Implications: This study points to chromosome $14 \mathrm{q} 32$ miRNAs as promising targets to inhibit tumor cell dissemination and to predict patient prognosis in lung adenocarcinoma. Mol Cancer Res; 16(3); 390-402. (O2018 AACR.

\section{Introduction}

Between $70 \%$ and $90 \%$ of lung cancer deaths are caused by metastases rather than by inability to control primary tumor growth (1), illustrating the need to understand molecular events that take place in different phases of tumor cell dissemination. Consequently, development of novel anticancer treatments focus- ing on inhibition of tumor metastasis could significantly improve patient outcome. It is crucial to identify robust tumor biomarkers that help to predict patients who are more likely to suffer from tumor cell dissemination and who will therefore benefit from therapeutic approaches targeting specific drivers of tumor cell metastasis.
'Institute of Pathology, University Hospital Heidelberg, Heidelberg, Germany. ${ }^{2}$ Systems Biology of Signal Transduction, German Cancer Research Center, Heidelberg, Germany. ${ }^{3}$ Division of Epigenetics, DKFZZMBH Alliance, German Cancer Research Center, Heidelberg, Germany. ${ }^{4}$ Center for Modeling and Simulation in the Biosciences (BIOMS), University of Heidelberg, Heidelberg, Germany. ${ }^{5}$ Life Sciences Research Unit, University of Luxembourg, Luxembourg, Luxembourg. ${ }^{6}$ Medical Research Centre, University of Heidelberg, Mannheim, Germany. ${ }^{7}$ Clinical Cooperation Unit Neuropathology, German Cancer Research Center (DKFZ), Heidelberg, Germany. ${ }^{8}$ Institute of Computational Biology, Helmholtz Center Munich, German Research Center for Environmental Health, Neuherberg, Germany. ${ }^{9}$ Department of Cancer Biology \& Genetics, Memorial Sloan Kettering Cancer Center, New York, New York. ${ }^{10}$ Institute of Medical Biometry and Informatics, University of Heidelberg, Heidelberg, Germany. ${ }^{11}$ Translational Lung Research Center Heidelberg (TLRCH), member of the German Center for Lung Research (DZL), Heidelberg, Germany. ${ }^{12}$ Translational Research Unit, Thoraxklinik at the University Hospital Heidelberg, Heidelberg, Germany. ${ }^{13} \mathrm{Helmholtz}$ University Group "Cell Plasticity and Epigenetic Remodeling," German Cancer Research Center, Heidelberg, Germany. ${ }^{14}$ Center for Computational and Theoretical Biology, University of Würzburg, Würzburg, Germany.

Note: Supplementary data for this article are available at Molecular Cancer Research Online (http://mcr.aacrjournals.org/)

Corresponding Author: Kai Breuhahn, University Hospital Heidelberg, Im Neuenheimer Feld 224, Heidelberg 69120, Germany. Phone: 49-6221-564675; Fax: 49-6221-56-5251; E-mail: kai.breuhahn@med.uni-heidelberg.de doi: 10.1158/1541-7786.MCR-17-0334

(c)2018 American Association for Cancer Research. 
In this regard, miRNAs are promising cancer biomarkers and represent target molecules for the development of new antitumor therapies. miRNAs are noncoding RNAs consisting of 1925 nucleotides that affect gene expression posttranscriptionally through the physical interaction with specific RNA transcripts (targets) leading to translational inhibition or degradation (2). Several studies have shown that miRNAs target oncogenes and tumor suppressor genes and, thus, are actively involved in regulating cancer hallmarks, such as proliferation, invasion, and metastasis (3). Given that individual miRNAs influence the bioavailability of hundreds of different mRNAs, they are able to control different cancer-related molecular pathways. This ability suggests that a miRNA-based therapy targeting key miRNAs or miRNA clusters will be less prone to develop drug resistance. In addition, miRNAs are very stable and detectable in tissues and biofluids (e.g., blood, serum, urine) with high sensitivity and specificity and, thus, can serve as useful biomarkers for early diagnosis, as well as prediction of prognosis and therapy response (4).

Although several miRNAs have been reported to be involved in lung carcinogenesis and progression (5), little is known about miRNAs involved in lung tumor dissemination. On the basis of high-throughput expression data derived from independent cohorts, we here aimed to identify key miRNAs associated with dissemination of lung adenocarcinoma, which is the most frequent primary lung cancer (6). We identified that upregulation of the $14 \mathrm{q} 32$ miRNA cluster was related to lymph node metastasis and a predictor of poor patient prognosis. In addition, we found that the overexpression of $14 \mathrm{q} 32$ miRNAs was associated with DNA hypomethylation. By applying the novel CRISPR activation (CRISPRa) technology, we demonstrated that coordinated activation of $14 \mathrm{q} 32$ miRNAs promoted cell migration and invasion. Finally, some first downstream targets of the identified miRNAs were characterized.

\section{Materials and Methods}

\section{Patient cohorts}

The discovery cohort consisted of a lung adenocarcinoma patient group from The Cancer Genome Atlas (TCGA) database. To avoid variability due to the use of different platforms, we selected only patients from which miRNA data from the Illumina HiSeq platform was available [https://tcga-data.nci.nih.gov/ (accessed in January 2015)]. The dataset included tumor samples from 449 lung adenocarcinoma patients, diagnosed from 1991 to 2013 , as well as 39 matched healthy tissues. The clinicopathologic characteristics of this patient cohort are shown in Supplementary Table S1 (Supplementary Material). In addition, mutation details of 181 patients out of this cohort were available on cBioPortal (http://www.cbioportal.org/).

The validation cohort consisted of an independent patient group that included 108 lung adenocarcinoma patients who underwent lung cancer resection from 2006 to 2011 at the Department of Thoracic Surgery of the Thoraxklinik at the University Hospital Heidelberg (Heidelberg, Germany). All samples were obtained with the informed consent of the patients and the approval of the local Ethics Committee of the University of Heidelberg (Heidelberg, Germany; 270/2001 and 206/2005). Each sample was snapfrozen in liquid nitrogen within 30 minutes after resection and stored at $-80^{\circ} \mathrm{C}$. Samples included tumor tissue from each patient $(n=108)$, as well as
68 matched healthy lung tissues. Clinical and histopathologic parameters were determined by clinicians and pathologists from the University Hospital Heidelberg (Supplementary Table S2; Supplementary Material).

\section{Methylation analysis}

Methylation analysis in lung adenocarcinoma tumors in comparison with healthy lung tissue was performed by using Illumina Infinium 450k DNA methylation microarray data from 60 patients of the discovery cohort ( 60 tumor samples and 27 healthy tissue samples; data downloaded from Genomic Data Commons database [https://gdc.cancer.gov/ (accessed on December 2016)]. The analysis was performed as described previously (7). In short, raw channel information was converted into methylation values and preprocessed in R using the Minfi library (SOURCE1; ref. 8). Preprocessing channel information consists of normalizing microarray data (SWAN method) and eliminating probes with a low detection significance $(P>0.01)$, location on XY-chromosomes, self-hybridization (as described in SOURCE2), and SNP contamination. Methylation values of the remaining probes were then extracted and analyzed for differential methylation. Finally, raw $P$ values were adjusted by a false discovery procedure (Benjamini-Hochberg) and filtered for statistical significance $(P \leq 0.05)$. To validate the results, 454 bisulfite sequencing (Roche) was used according to the manufacturer's instructions for the analysis of DNA methylation at specific genomic regions of sample pools of healthy lung tissues $(n=18)$, tumors from patients without lymph node metastasis (No stage; $n=19$ ), and tumors from patients with lymph node metastasis $(\mathrm{N} \geq 1$ stage; $n=24)$. Primer sequences for 454 bisulfite sequencing are listed in Supplementary Table S3 (Supplementary Material).

\section{Additional information}

Detailed information on the methods used for nucleic acid isolation, qRT-PCR, Western blotting, cell culture and transfection, CRISPRa technology, viability assays, migration and invasion assays, Sanger bisulfite sequencing, microarray analysis, as well as statistical analysis can be found in Supplementary Materials and Methods as well as Supplementary Tables S4-S7.

\section{Results}

Overexpression of miRNAs located on 14q32 is associated with metastasis in lung adenocarcinoma patients

To identify miRNAs involved in lung adenocarcinoma cell dissemination, we analyzed sequencing data from a TCGA patient cohort containing 449 tumor samples and 39 matched healthy lung tissue samples (discovery cohort). Candidate miRNAs associated with lymph node metastasis were selected according to three criteria: (i) differential expression in patients with lymph node metastasis ( $\mathrm{N} 1-3$ stages, hereafter indicated as $\mathrm{N} \geq 1$ ) versus patients without lymph node metastasis (N0 stage); (ii) significant expression changes in tumors compared with healthy tissue; (iii) high expression variability among the tumors ( $1 / 3$ top of the miRNA list ranked by the expression variance; Fig. 1A). Fifty-one miRNAs accomplished these criteria (intersection area of graph), and we selected the 28 of them that were dysregulated in the same direction ( 2 downregulated and 26 upregulated) in both comparisons (i.e., tumor vs. healthy tissue and $\mathrm{N} \geq 1$ stage vs. N0 stage). Interestingly, 16 of these 28 candidates (57.1\%) were located on the same genomic region on chromosome 14q32 (Fig. 1B). 
A

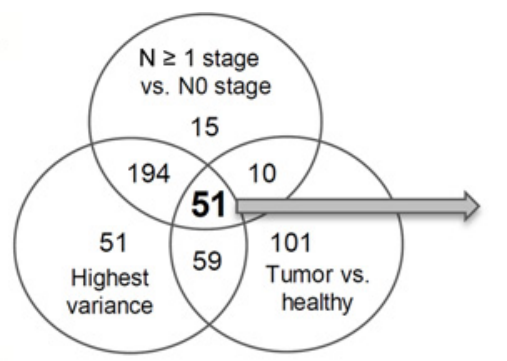

C
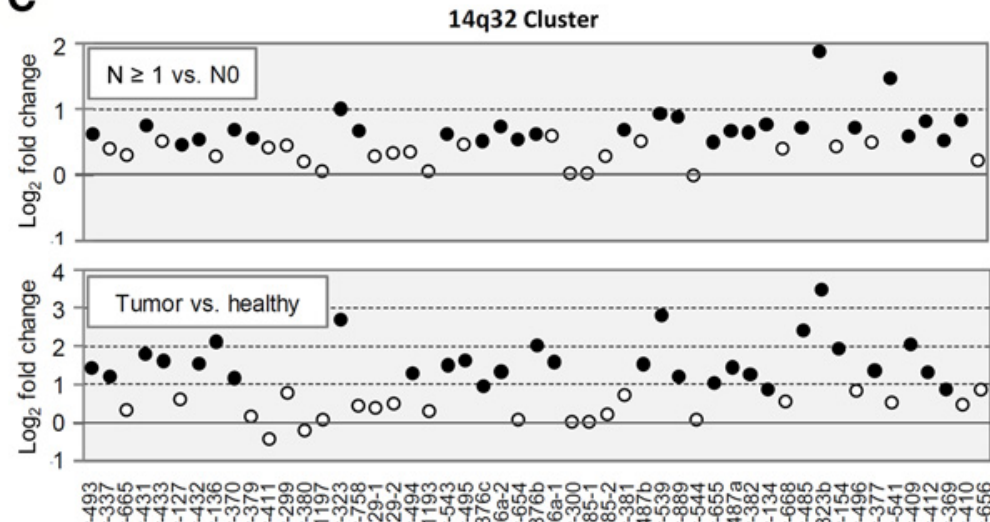

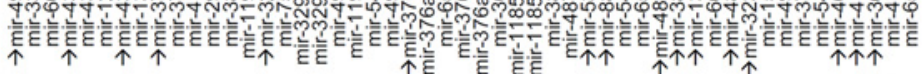

D

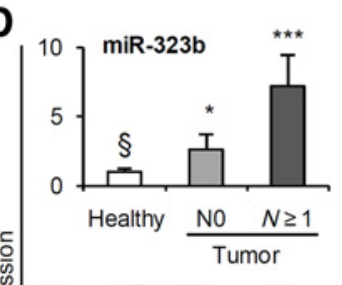

E
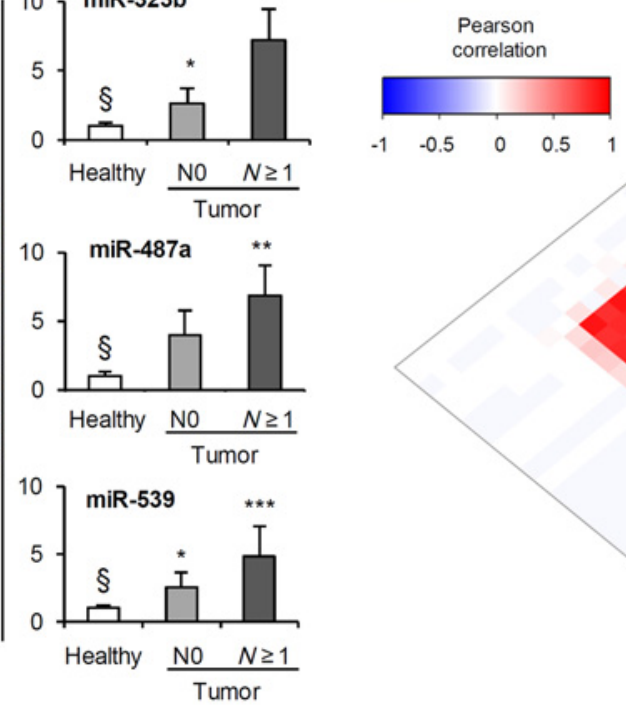

$14 \mathrm{q} 32$ miRNA section

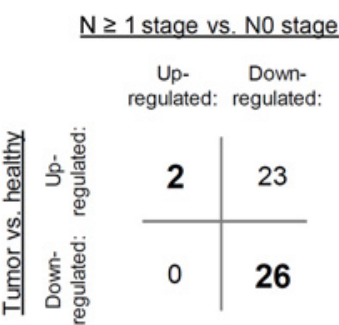

B

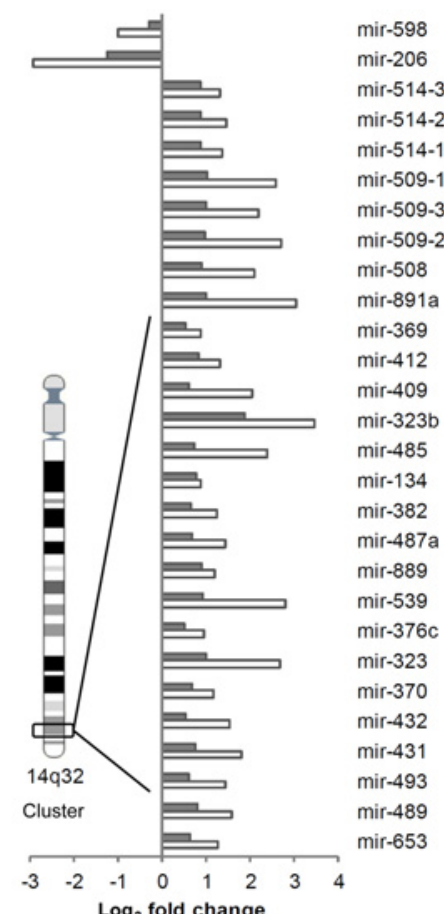

$\mathbf{F}$
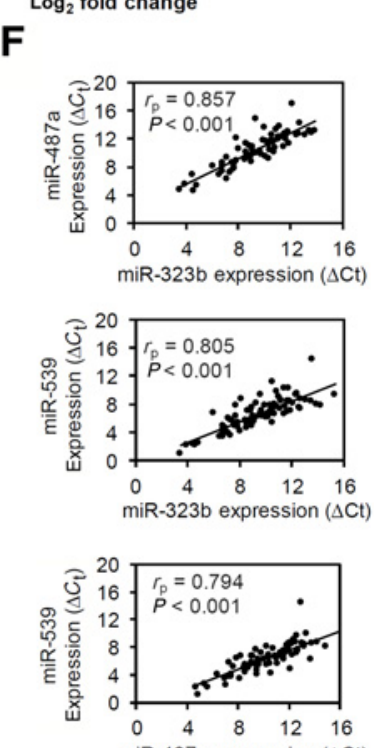

Figure 1.

Dysregulated miRNAs in lung adenocarcinoma patients with lymphatic metastasis. A, Venn diagrams illustrating the selection of miRNAs involved in lung adenocarcinoma metastasis from TCGA sequencing data. Selection criteria included differential expression in tumors ( $n=449)$ compared with healthy tissue $(n=39)$, in patients with lymph node metastasis ( $\mathrm{N} \geq 1$ stage) compared with patients without lymph node metastasis (NO stage), and high expression variance among tumors (top 1/3). EdgeR-GLM algorithm was applied to analyze miRNA differential expression. miRNAs modulated in the same direction in both comparisons (tumors vs. healthy; $\mathrm{N} \geq 1$ stage vs. NO stage) were selected to be potentially involved in tumor spread. B, Differential expression levels of the 28 selected miRNAs (FDR $\leq 0.05$; Benjamini-Hochberg method); among them, 16 (57\%) were located on the $14 \mathrm{q} 32$ cluster. C, Differential expression of all miRNAs located on the $14 \mathrm{q} 32$ cluster in lung adenocarcinoma tumors versus normal tissue, and $\mathrm{N} \geq 1$ stage versus $\mathrm{NO}$ stage patients. Black dots represent statistically significant results (FDR $<0.05)$. miRNAs selected in the previous analysis as potentially involved in tumor dissemination are highlighted with arrows. D, Validation of three selected miRNAs by qRT-PCR in an independent lung adenocarcinoma patient cohort $(n=108)$. Results were analyzed by the $2^{-\Delta \Delta C_{t}}$ method and bars represent mean \pm SEM. Mann-Whitney test was used for statistical comparison $\left({ }^{*}, P \leq 0.05 ;{ }^{* *}, P \leq 0.01 ;{ }^{* * *}, P \leq 0.001 ; \S\right.$ control). E, Correlation plot of the 28 selected miRNAs potentially involved in lymph node metastasis in lung adenocarcinoma patients (TCGA cohort; $n=449$ ). F, Validation of the correlation of miR-323b, miR-487a, and miR-539 expressions by qRT-PCR in an independent lung adenocarcinoma cohort $(n=108) . r_{\mathrm{p}}$ : Pearson correlation coefficient. 
Moreover, the majority of the 52 miRNAs located on this region (14q32 miRNA cluster) were significantly overexpressed in both tumors versus healthy tissue (56.9\%), as well as in $\mathrm{N} \geq 1$ stage compared with N0 stage patients (54.9\%; Fig. 1C). Remarkably, candidate miRNAs (arrows in Fig. 1C) are distributed at a higher density on a specific region of the cluster ranging from mir-539 to mir-369 (referred to as "14q32 miRNA section," Fig. 1C).

Overexpression of these $14 \mathrm{q} 32$ miRNAs in lung adenocarcinoma patients with lymph node metastasis was validated by qRT-PCR in an independent lung adenocarcinoma patient cohort containing 108 fresh-frozen tumor samples and 68 matched healthy lung tissue samples (validation cohort). Three candidate miRNAs from the selected $14 \mathrm{q} 32$ cluster were chosen for validation: mir-323b and mir-539, which showed the highest differential expression in $\mathrm{N} \geq 1$ stage patients; as well as mir487 a, which was previously reported to be involved in metastasis of other cancer types $(9,10)$. The expression of the mature (functional) forms of these miRNAs (miR-323b-3p, miR-5395p, and miR-487a-3p; hereinafter called miR-323b, miR-539, and miR-487a, respectively) gradually increased from healthy tissue through tumors without metastasis to tumors with lymph node metastasis (Fig. 1D). The expression of these miRNAs was not associated to the mutational status of EGFR or TP53, but mir-539 showed a significant decrease in KRASmutated tumors (Supplementary Fig. S1). Moreover, the analysis of never-smoker patients showed that mir-487a significantly increased in tumors with EGFR mutations (Supplementary Fig. S1). On the other hand, a significant coexpression of the clustered miRNAs was observed in tumors of the discovery cohort (Fig. 1E), and coexpression of the selected 14q32 miRNAs was confirmed in the validation cohort (Fig. 1F).

These results show a coordinated and progressive increase in 14q32 miRNAs from healthy tissue to metastatic disease, which suggests that they may play a functional role in lung adenocarcinoma cell dissemination and that a common molecular mechanism may facilitate their dysregulation in tumor cells.

Hypomethylation of the 14q32 genomic region is associated with overexpression of metastasis-related miRNAs

Disruption of a normal epigenetic regulation is a prominent feature of human cancers (11). In terms of DNA methylation, arguably the most extensively studied epigenetic mechanism, tumor cells frequently display a global hypomethylation of lamina-associated domains together with a widespread CpG island promoter hypermethylation (12). Considering that these aberrations are known to have a profound impact on gene expression, we decided to investigate whether DNA methylation changes could be the molecular reason for the coordinated overexpression of the identified miRNAs. First, we analyzed DNA methylation microarray TCGA data of 27 healthy tissue samples and 60 tumor samples randomly selected from the discovery cohort. Confirming our hypothesis, we found a significant global hypomethylation of the 14q32 miRNA locus, which is part of the DLK1-DIO3 cluster $(13,14)$, in tumors compared with healthy lung tissue (Fig. 2A).

To confirm these results, 61 DNA samples from the validation cohort, including $24 \mathrm{~N} \geq 1$ stage tumors, 19 N0 stage tumors, and 18 healthy tissues, were analyzed for their methylation status by bisulfite sequencing. The genomic regions covering the locations of mir-323b, mir-487a, and mir-539 were chosen for this analysis.
The results confirmed genomic hypomethylation in tumors compared with healthy tissues and showed that methylation was lower in tumors with lymph node metastasis compared with tumors without cancer cell dissemination (Fig. 2B). Moreover, real-time PCR analysis revealed an association between mir-323b, mir-487a, and mir-539 levels and increased genomic hypomethylation of the $14 \mathrm{q} 32$ locus from healthy lung tissue to tumors from patients with lymph node metastasis (Fig. 2C).

The DLK1-DIO3 cluster is an imprinted domain known to be mainly regulated by two differentially methylated regions (DMR), the intergenic DMR (IG-DMR), located between the DLK1 and MEG3 genes, and the MEG3-DMR, comprising the promoter and beginning of MEG3 (15). As cancer-related loss of imprinting (LOI) of the DLK1-DIO3 domain had previously been associated with an overexpression of its miRNAs (16), we also investigated the methylation status of IG-DMR and MEG3-DMR in lung adenocarcinoma. Although our TCGA data analysis comparing 27 healthy and 60 tumor samples showed a potential deregulation in these regions (Fig. 2A), the same comparison using $60 \mathrm{NO}$ and $60 \mathrm{~N} \geq 1$ stage tumors did not reveal any significant changes (data not shown). We confirmed the unchanged methylation status of three representative regions within IG-DMR and MEG3-DMR in healthy, N0, and $\mathrm{N} \geq 1$ stage patients, using Sanger bisulfite sequencing (Supplementary Fig. S2). Altogether, our results revealed that a global epigenetic deregulation of the DLK1-DIO3 locus in lung adenocarcinoma cells with specific hypomethylation of its miRNA clusters associates with their overexpression in the context of metastasis and tumor cell dissemination.

\section{Expression levels of individual 14q32 miRNAs are potential} prognostic markers in never-smoker patients

To study the potential value of the selected $14 \mathrm{q} 32$ miRNAs (mir-323b, mir-487a, and mir-539) as prognostic cancer biomarkers, we determined their association with patient disease-free survival (DFS) in the discovery cohort by applying Kaplan-Meier analysis. We found a significant association of mir-539 overexpression and poor patient DFS by using the median expression value as cutoff (Fig. 3). The association with mir-487a and mir$323 \mathrm{~b}$ was only significant when the best cutoff was applied (for median as cutoff: $P=0.6$ and $P=0.5$, respectively). To explore the possible differences according to patient smoking history, Kaplan-Meier analyses were performed after respective patient stratification. Interestingly, overexpression of the three miRNAs independently was significantly associated with a shorter time to relapse in never-smoker patients $(n=47)$, whereas this association was not found in smokers at the time of diagnosis (current smokers, $n=78$ ). Remarkably, although 14q32 miRNA levels were related to lymph node metastasis ( $\mathrm{N}$ stage) in lung adenocarcinoma patients, association of DFS with $\mathrm{N}$ stage in neversmoker patients did not reach the level of significance $(P=0.09$; Supplementary Fig. S3), indicating that miRNA levels could be more relevant to predict patient prognosis than lymph node metastasis in this patient group.

The association of DFS and miRNA expression was also assessed by proportional hazards Cox regression modeling in the group of never-smoker patients. Univariate analysis showed that higher expressions of mir-539, mir-487a, and mir-323b were significantly associated with increased risk of lung adenocarcinoma relapse compared with lower miRNA expression (Fig. 3). The association of clinicopathologic parameters and 
González-Vallinas et al.

A

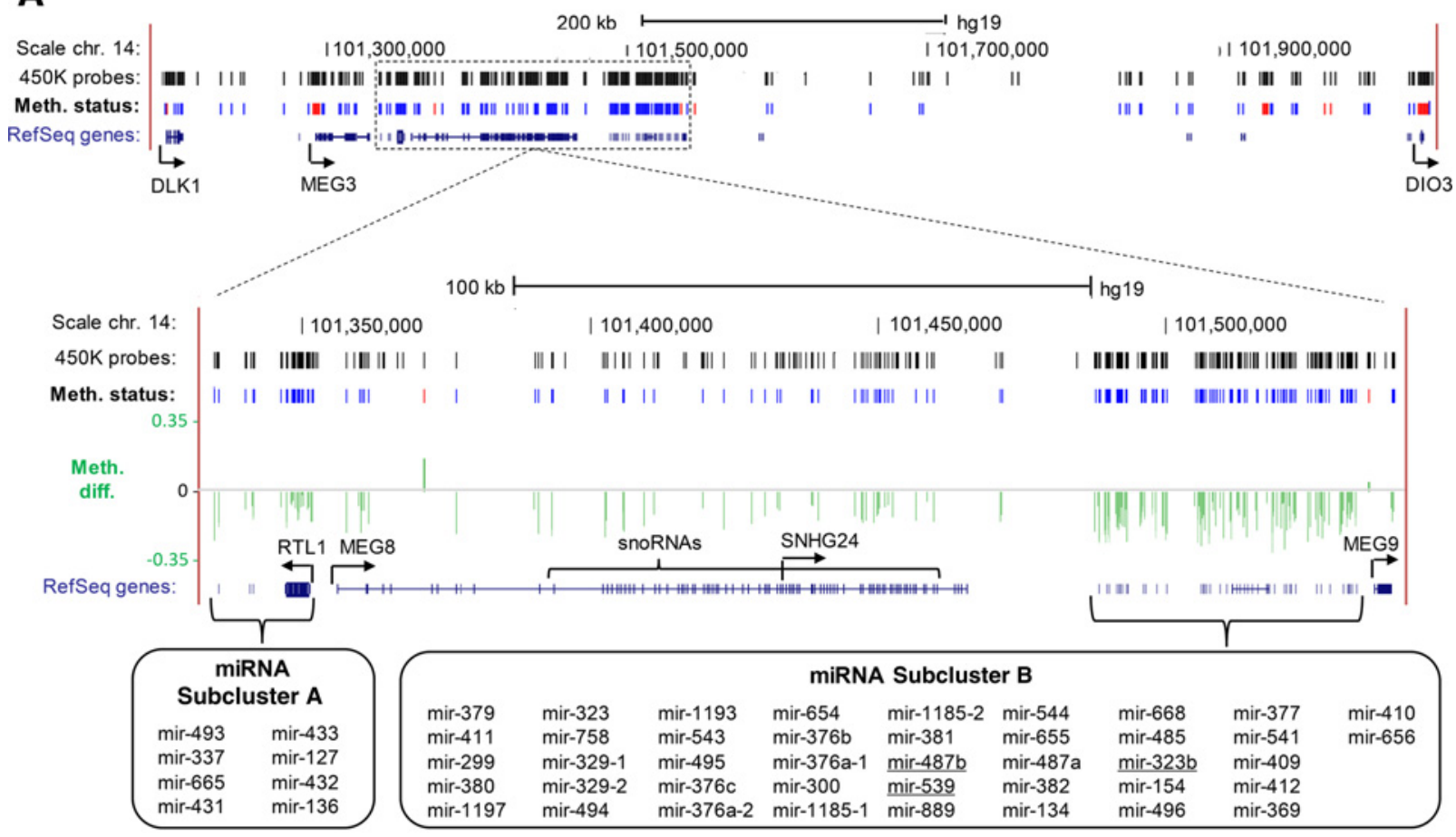

B
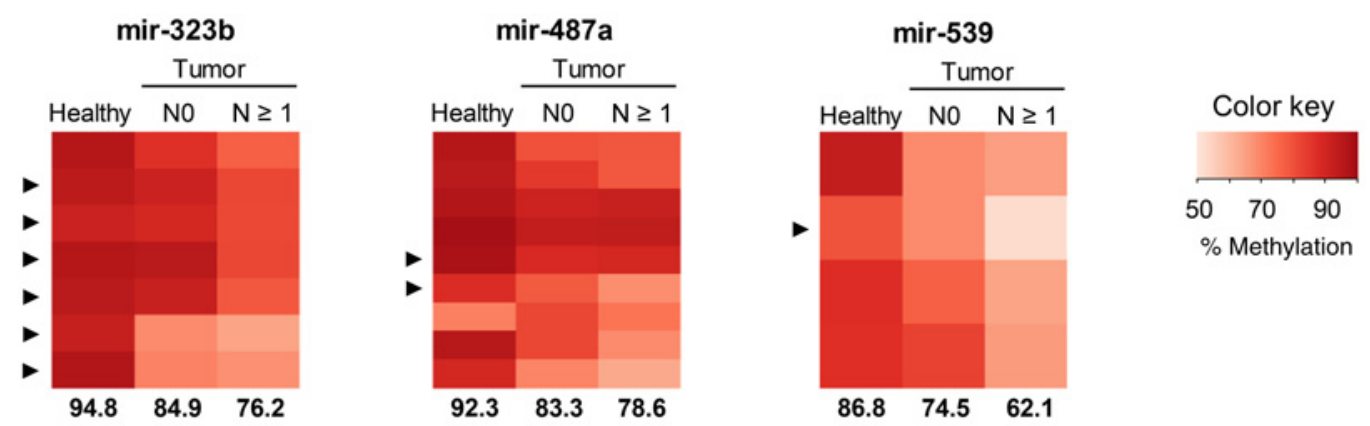

C
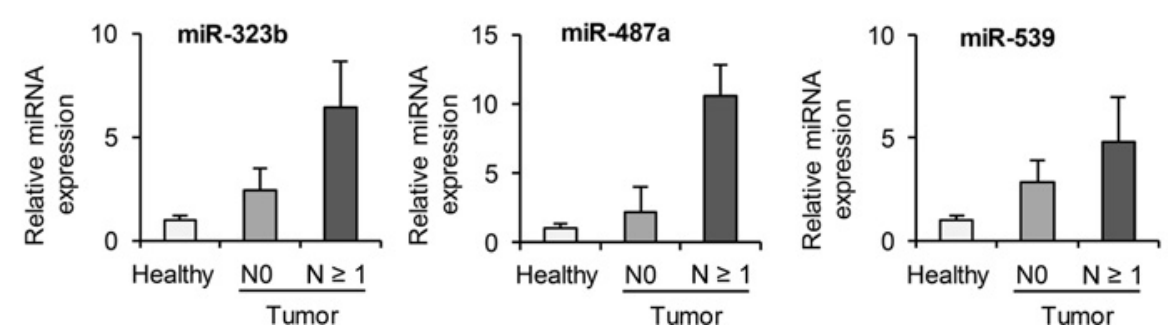

Figure 2.

Hypomethylation of the $14 \mathrm{q} 32$ locus is associated with overexpression of the clustered miRNAs in disseminated lung adenocarcinoma. A, Methylation analysis of the DLK1-DIO3 cluster, which contains the 14q32 miRNA cluster consisted of two miRNA subclusters, in lung adenocarcinoma tumors $(n=60)$ compared with healthy lung tissue $(n=27)$ by using Illumina Infinium 450k DNA methylation microarray data of a patient cohort from the Genomic Data Commons database. Methylation status: red, hypermethylated; blue, hypomethylated. Meth. diff.: $\beta$-value of the differential methylation between tumor and healthy lung tissue. 450k probes indicate the $\mathrm{CpGs}$ analyzed in the arrays and RefSeq genes row shows the location of known transcripts. Brackets indicate the regions of miRNAs within this genomic locus. B, Validation of the differential DNA methylation in an independent patient cohort by 454 bisulfite sequencing. Samples consisted of pools of healthy lung tissue $(n=18)$, tumor tissue from No stage patients $(n=19)$, and tumors from patients with lymph node metastasis ( $\mathrm{N} \geq 1$ stage; $n=24$ ). Genomic regions of $14 q 32$ covering the location of mir-323b, mir-487a, and mir-539 were selected for the validation. The CpGs located within the respective miRNA locus are indicated by black arrowheads. Numbers below the panels indicate the average of the methylation percentages of the different CpG sites in each sample type. C, Expression levels of miR-323b, miR-487a, and miR-539 in the lung adenocarcinoma patients analyzed in $\mathbf{B}$. Results were calculated by the $2^{-\Delta \Delta C_{t}}$ method and bars represent mean \pm SEM. 
Complete cohort
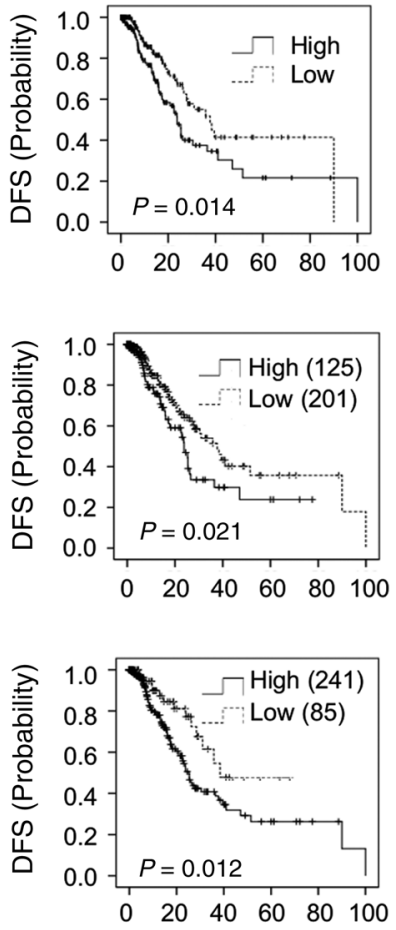
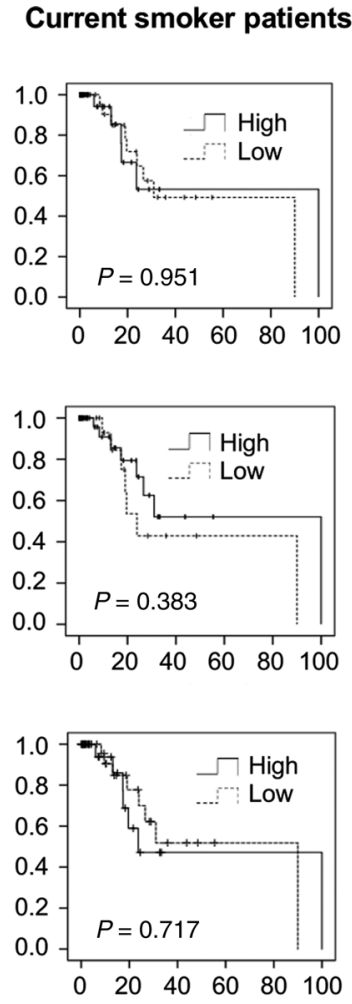

Never-smoker patients
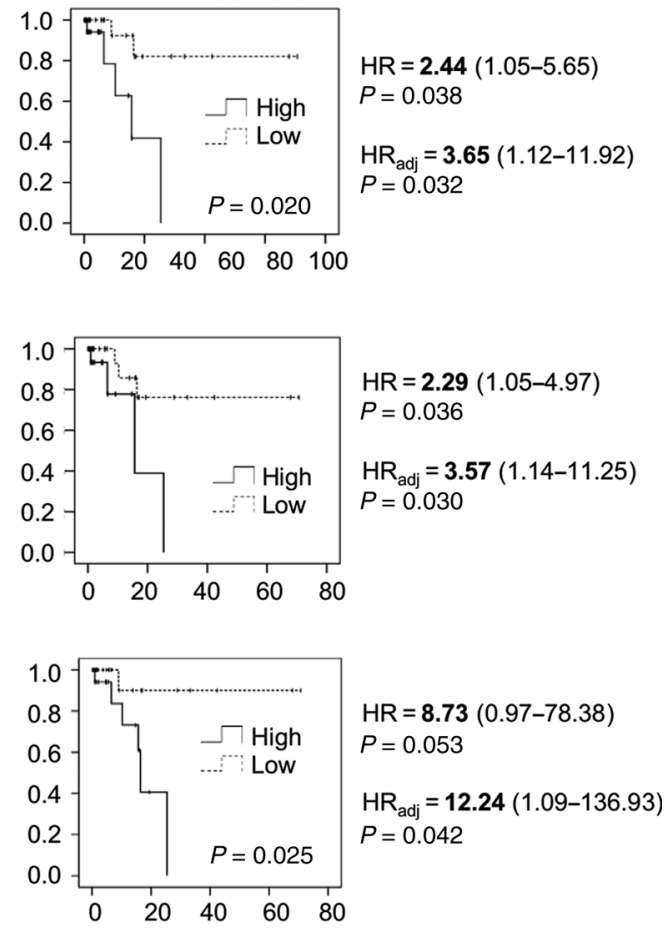

Time (months)

Figure 3.

Expression of mir-323b, mir-487a, and mir-539 is associated with DFS in never-smoker lung adenocarcinoma patients. Kaplan-Meier analyses of selected 14 q32 miRNAs in DFS of lung adenocarcinoma patients (complete cohort and stratified by smoking status). Sequencing data from the TCGA lung adenocarcinoma patient cohort $(n=449)$ was used to determine miRNA expression levels (high/low). Median of expression was used as cutoff, unless the number of patients in each group is indicated. Because of the exploratory character of this analysis, the results were not adjusted for multiple testing (46). The log-rank test was applied to calculate $P$ values of Kaplan-Meier curves. On the right, HRs and $95 \%$ confidence lintervals ( $95 \% \mathrm{Cl}$ ) for the association of DFS and miRNA expression in never-smokers are shown. Values were calculated by univariate proportional hazards Cox regression modeling (HR) and adjusted for $\mathrm{N}$ stage, $\mathrm{T}$ stage, and cancer stage in a multiple Cox proportional hazard regression (HRadj).

patient DFS was determined by univariate analysis and pointed to T stage $(P=0.024), \mathrm{N}$ stage $(P=0.013)$, and cancer stage $(P=0.003)$ as potential confounding factors $(P \leq 0.05)$. Multiple Cox analysis showed that the statistical association between miRNA expression and DFS was increased after correcting for these confounding factors (Fig. 3), indicating that miRNA expression may represent an independent prognostic biomarker in never-smoker lung adenocarcinoma patients. In summary, these results indicate that mir-539, mir-323b, and mir-487a may serve as prognostic biomarkers for lung adenocarcinoma patients with highest relevance for never-smokers.

Coordinated overexpression of 14q32 miRNAs increases cell migration and invasion

The analysis of dysregulated miRNAs in lung adenocarcinoma samples with lymph node metastasis illustrated that a specific section of the 14q32 miRNA cluster (14q32 miRNA section: from mir-539 to mir-369) contained a high proportion of miRNAs associated with lymph node metastasis (Fig. 1C). To further investigate the functional relevance of these 14q32 miRNAs for tumor cell migration and invasiveness, we applied CRISPR-Cas9based gene activation technology (CRISPRa) using a mutant Cas9 lacking endonuclease activity (dCas9) fused with the activating domains VP64, p65, and Rta (VPR; refs. 17, 18). To coordinately upregulate the specific 14q32 miRNA section (which contained the selected miRNAs mir-323b, mir-487a, and mir-539), we designed single-guide RNAs (sgRNA) that bound the genomic region upstream of the transcription starting site of mir-539 (Fig. 4A). Five different sgRNAs were tested in the lung adenocarcinoma cell line $\mathrm{H} 2009$ stably expressing dCas9-VPR (Fig. 4B). miRNA expression analysis by qRT-PCR revealed that all tested sgRNAs successfully induced expression of miR-323b, miR-487a, and miR-539, which were part of the 14q32 miRNA section (Fig. 4C). Cells transduced with sgRNA-A and sgRNA-B (CRISPRa-14q32 cells), which showed the highest miRNA induction, were selected for further functional experiments.

To analyze the biological relevance of coordinated $14 \mathrm{q} 32$ miRNA expression in tumor cell migration, we performed lateral migration experiments with predefined gaps. The directed cell mobility was monitored by time-lapse microscopy. CRISPRa-14q32 cells stably expressing either sgRNA-A or sgRNA-B possessed significantly increased lateral migration ability (approximately 80\%-90\% increase) compared with CRISPRa-control cells, which were transduced with the empty 
González-Vallinas et al.

A
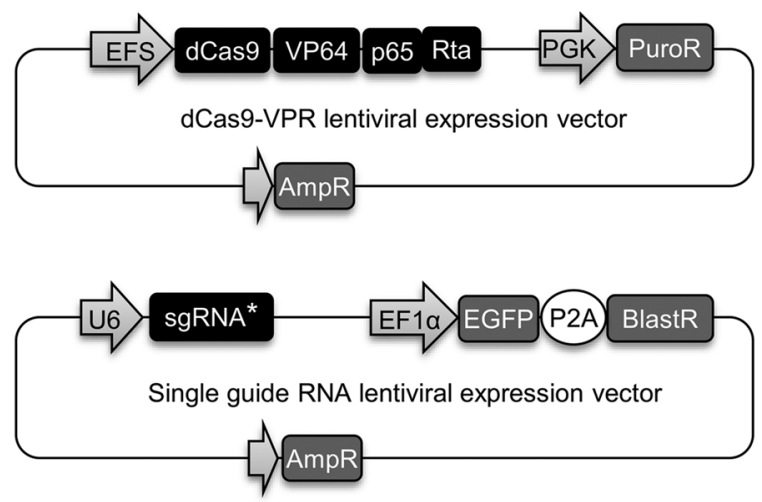

B

* Five different sgRNAs - Location:

C

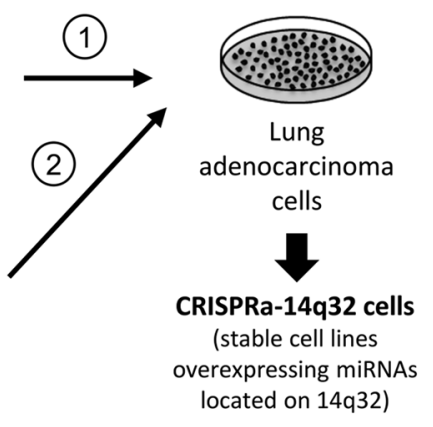

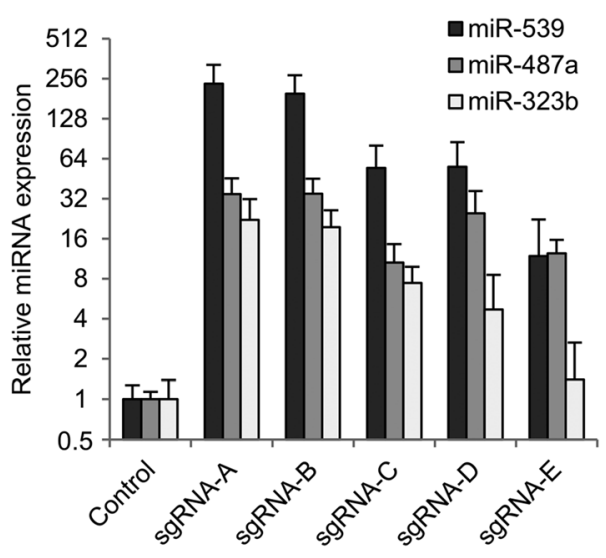

$\mathbf{E}$

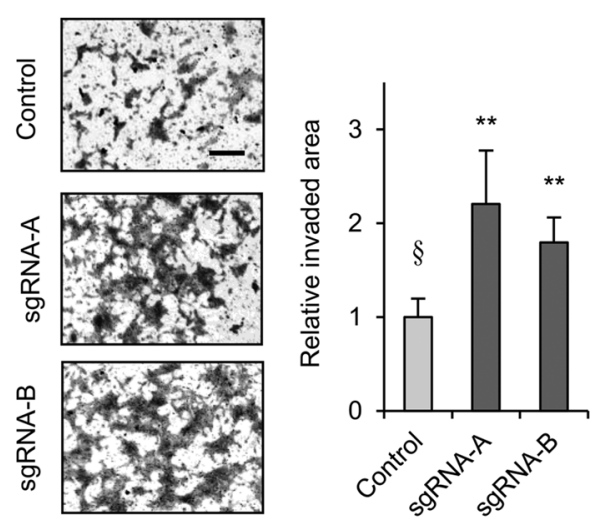

Figure 4.

Upregulation of 14q32 miRNAs by CRISPR-dCas9 technology induces migration and invasion of lung adenocarcinoma cells. A, Simplified layout of the lentiviral expression vectors used for stable activation of $14 \mathrm{q} 32$ miRNAs. First vector encodes a mutant Cas 9 with undetectable endonuclease activity (dCas9) fused with the activating domains VP64, p65, and Rta (VPR). Second vector encodes the specific sgRNA. Promoters are depicted with arrows. B, Genomic binding sites of the 5 different sgRNAs designed. C, Overexpression of selected 14q32 miRNAs in the five different CRISPRa-14q32 cell lines determined by qRT-PCR. Control cells are generated by transduction of both dCas9-VPR and sgRNA vectors without including sgRNA sequence. D, Cell migration of two different CRISPRa-14q32 clones compared with controls over a $500-\mu \mathrm{m}$ gap area for 12 hours. The graph shows quantification of four independent experiments. E, Invasion of CRISPRa-14q32 cells through a Matrigel basement membrane matrix after 48 hours. The graph shows quantification of three independent experiments. Student $t$ test was used for statistical comparison $\left(^{*}, P \leq 0.05\right.$; ${ }^{* *}, P \leq 0.01 ;{ }^{* * *}, P \leq 0.001 ; \S$ control). Scale bar, $500 \mu \mathrm{m}$. 
sgRNA vector (Fig. 4D). In addition, invasion analysis using Matrigel invasion chambers showed that $14 \mathrm{q} 32$ miRNA overexpression also conferred a significant increase (approximately double) in cell invasiveness compared with controls (Fig. 4E). Importantly, cell viability was not affected by $14 \mathrm{q} 32$ miRNA cluster overexpression, indicating that changes in cell numbers did not account for the observed migratory/invasive phenotype (Supplementary Fig. S4).

Together, these experiments demonstrate that the dysregulation of $14 \mathrm{q} 32$ miRNAs is functionally associated with a prometastatic phenotype of lung cancer cells.

\section{Modulation of genes involved in cell motility processes} explains the biological effects of $14 \mathrm{q} 32$ miRNAs

To decipher molecular mechanisms underlying the role of the $14 \mathrm{q} 32$ miRNAs in lung adenocarcinoma dissemination, we performed transcriptome analysis of the two clones of CRISPRa$14 \mathrm{q} 32$ cells (sgRNA-A or sgRNA-B) in comparison with CRISPRacontrol cells. The results of the array analysis confirmed that levels of genes/lncRNAs adjacent to the overexpressed miRNA cluster were not modified in CRISPR-14q32 cells compared with control cells (Supplementary Fig. S5), indicating that potential phenotypic differences between these cell lines are exclusively due to the specific miRNA overexpression. In total, 352 genes were significantly and commonly regulated in both cell lines, among which 197 were downregulated and 155 were upregulated (Fig. 5A). Subsequent Gene Ontology (GO) enrichment analysis identified eight biological processes significantly represented in this gene list (Fig. 5B). Interestingly, the four processes with the highest level of significance were related to cell movement (i.e., cell motility, movement of cell or subcellular component, cell migration, locomotion), supporting involvement of the 14q32 miRNA section in tumor cell dissemination. Among the significantly modulated genes, 50 (14.2\%) were involved in these four migration-related processes (Fig. 5C). To validate these results, seven genes were selected on the basis of their association with metastasis and/or poor survival in lung cancer and other tumor types. Low levels of EPHA4 and OVOL2 have been associated with poor survival and epithelial-to-mesenchymal transition (EMT), respectively, in lung adenocarcinoma $(19,20)$. On the other hand, increased expressions of LOXL2, $A X L$, and $C D H 2$ have been associated with poor prognosis in lung cancer (21-23), whereas high levels of the kinesin family members KIF3C and KIF20A were associated with lymph node metastasis in breast cancer and with poor overall survival in cervical squamous cell carcinoma, respectively $(24,25)$. qRT-PCR analyses confirmed downregulation of OVOL2 and EPHA4 and upregulation of LOXL2, AXL, CDH2, KIF3C, and KIF20A in the CRISPRa-14q32 cells (Fig. 5D). Moreover, the same dysregulation of these genes was observed to be associated with poor DFS in lung adenocarcinoma patients from the discovery cohort (Supplementary Fig. S6), supporting their relevance in tumor development.

Altogether, these results indicate that $14 \mathrm{q} 32$ miRNAs modulate several metastasis-related genes, which may be involved in the increased invasion and migration capacity of lung adenocarcinoma cells.

miR-323b, miR-487a, and miR-539 individually promote tumor cell migration and invasion

In the next step, we analyzed the biological impact of individual miRNAs on cell migration and invasiveness. Because we expected much weaker functional effects of single miRNAs and because endpoint assays may not capture the dynamic properties of cell mobility (e.g., velocity, temporary changes in directional movement), we decided to utilize a method for quantitative analysis of lateral cell migration by time-lapse microscopy based on particle image velocimetry (26).

For these experiments, we selected the most promising miRNA candidates from the $14 \mathrm{q} 32$ cluster (miR-323b and miR-539, which exhibited the highest expression in $\mathrm{N} \geq 1$ vs. No stages; and miR-487a, previously associated with metastasis in other tumor types; refs. 9, 10) and transfected respective miRNA mimics in $\mathrm{H} 2009$ cells. A close-meshed analysis of cell movement revealed that all analyzed miRNAs significantly increased lateral migration of transfected cancer cells in comparison with control cells (Fig. 6A). Interestingly, none of the three miRNAs affected very early phases of cell migration (up to 20 hours) but led to a higher average speed of the cancer cells (Fig. 6B). All miRNAs increased cell velocity after 20 to 70 hours in a comparable manner. Notably, individual miRNAs did not change cell viability up to 72 hours after transfection, demonstrating that proliferation did not affect the experiment (Supplementary Fig. S7). Moreover, we examined the influence of individual miRNAs on cell invasiveness. The results illustrated that all tested miRNAs significantly supported tumor cell invasion. Interestingly, miR-487a and miR-539 were the strongest inducers of cell invasion, whereas miR-323b moderately increased invasiveness (Fig. 6C).

To determine migration-related genes underlying the observed biological effects of individual miRNAs, we analyzed the expression of the genes previously found to be modulated in the CRISPRa-14q32 cells after transfection with the mimics specific for miR-323b, miR-487a, and miR-539 (Fig. 6D). The results showed that all three miRNAs strongly induced $A X L$. In addition, miR-539 moderately decreased EPHA4 and increased KIF20A, whereas miR-487a significantly reduced the levels of OVOL2. Among the downregulated genes, EPHA4 was predicted to be a direct target of miRNA-539 according to TargetScan. However, the direct interaction was not confirmed by luciferase assay (data not shown). Importantly, most of the genes involved in cell movement that were modulated by the miRNAs showed increase levels when the miRNAs were overexpressed (e.g., KIF20A, AXL), suggesting that they are indirectly regulated by the miRNAs.

In summary, the results illustrate that miR-323b, miR-487a, and miR-539 individually support lung adenocarcinoma cell dissemination by the modulation of migration-related genes through different mechanisms. Additional cell lines with different KRAS and EGFR mutation status were used to validate the effects of the miRNAs regarding migration, invasion, and AXL expression. The results exhibit that each miRNA induced AXL expression, both at gene and protein levels, in every lung adenocarcinoma cell line and increased cell motility in at least two different cell lines (Supplementary Fig. S8).

\section{Discussion}

Development of local and distant metastasis is a critical event defining lung cancer patient survival (27). Accordingly, lymph node metastasis is one of the most important factors in determining lung cancer treatment, illustrating the necessity 
A

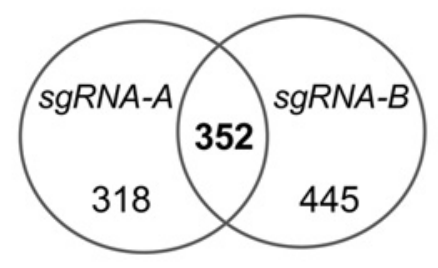

C
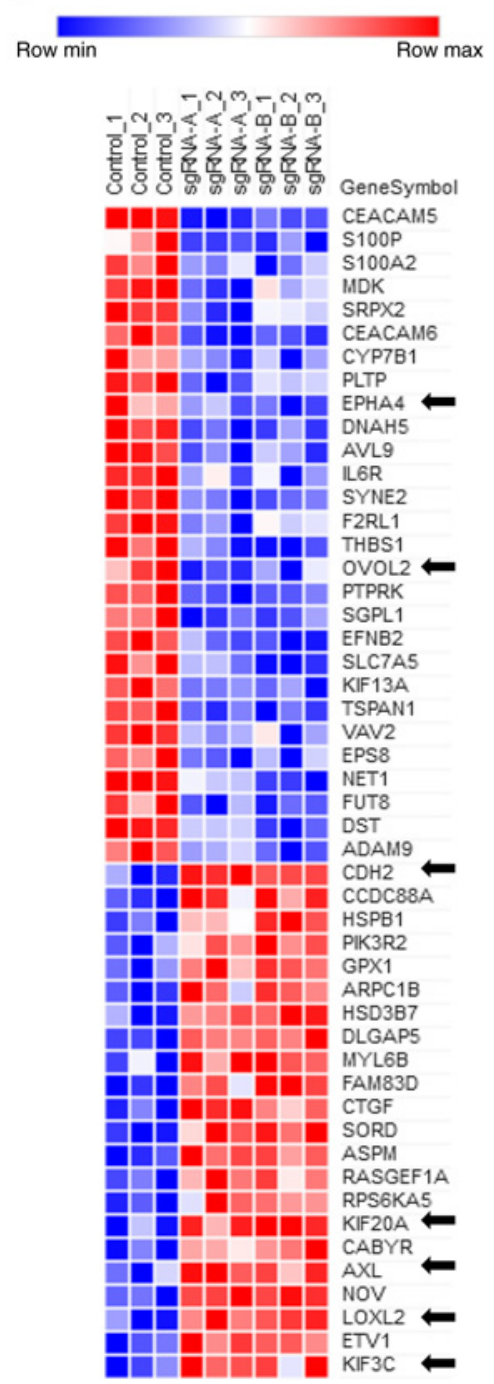

B

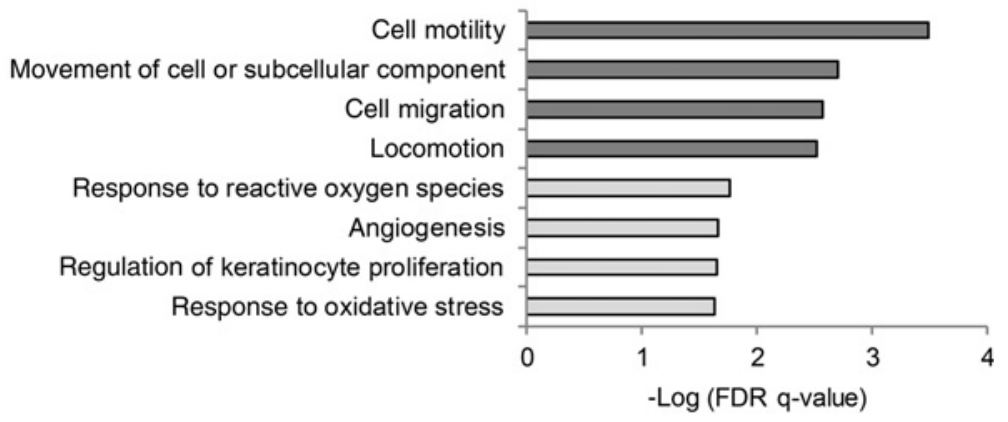

EPHA4

OVOL2

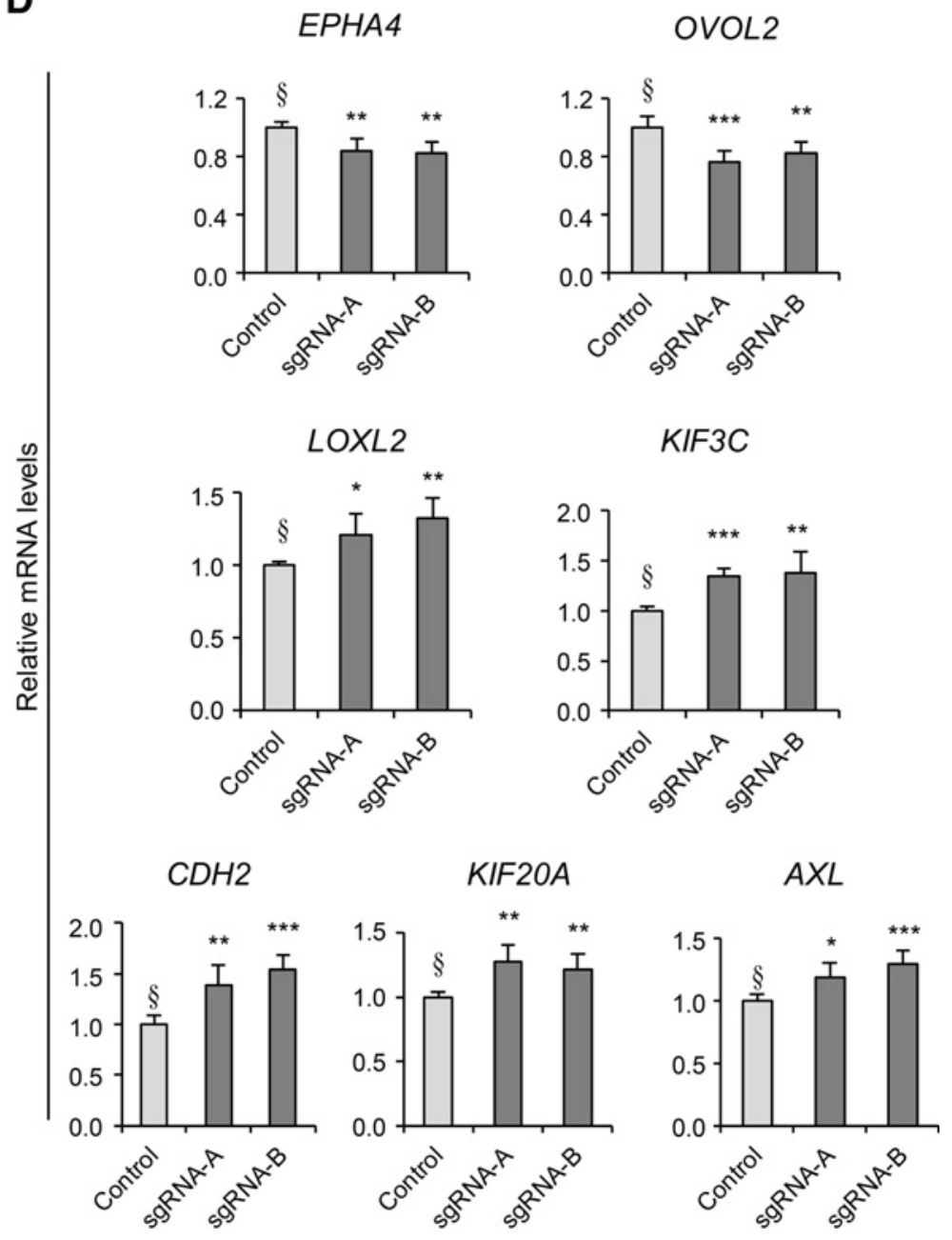

$\mathrm{CDH} 2$

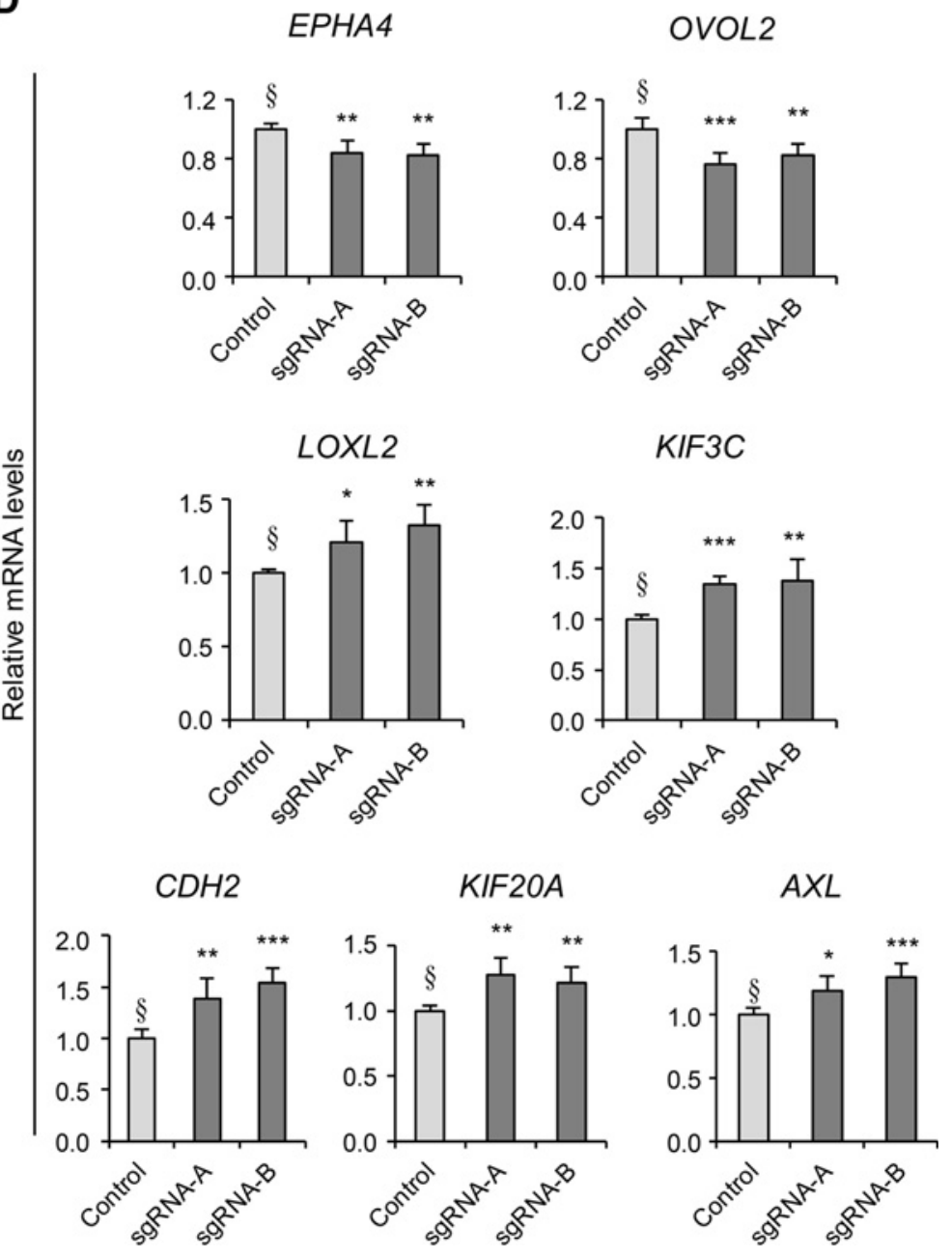

Biological process

D

Figure 5.

Modulation of gene expression by 14q32 miRNAs. A, Venn diagram showing the significantly modulated genes in two different CRISPRa-14q32 cell lines (sgRNA-A and sgRNA-B) compared with CRISPRa-control cells determined by whole-genome expression microarray analysis. B, GO analysis performed with the 352 commonly regulated genes revealed the significant overrepresentation of four biological functions related to cell movement (dark gray bars). The graph shows the functions with fold enrichment $>2$ and FDR q-value $<0.05$. C, Heatmap exhibiting the 50 significantly modulated genes (FDR $\leq 0.05$ ) in both sgRNA-A and sgRNA-B CRISPR-14q32 clones involved in the migration-related biological functions determined in B. D, Validation of selected migration-related genes in CRISPRa-14q32 cells by qRT-PCR. Expression levels were analyzed by the $2^{-\Delta \Delta c_{t}}$ method. Student $t$ test was used for statistical comparison $\left({ }^{*}, P \leq 0.05 ;{ }^{* *}, P \leq 0.01 ;{ }^{* *}, P \leq 0.001 ; \S\right.$ control). 
A

Control
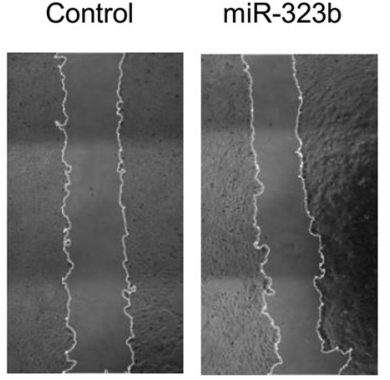

miR-487a
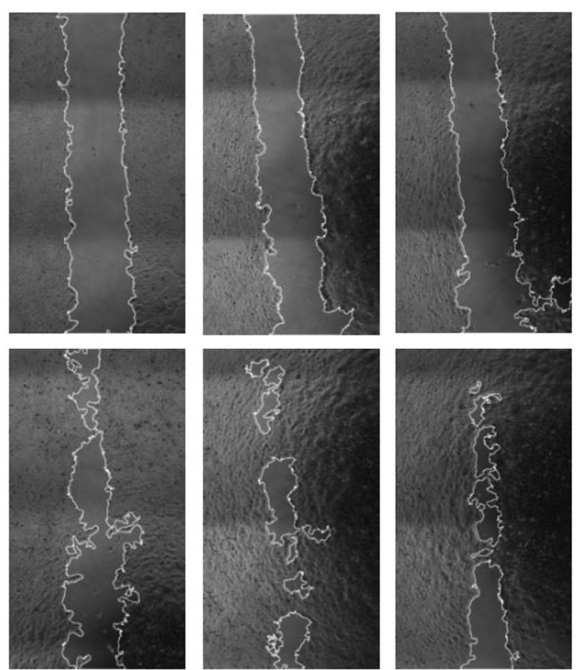

miR-539
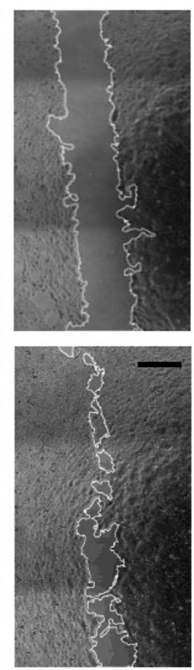

B

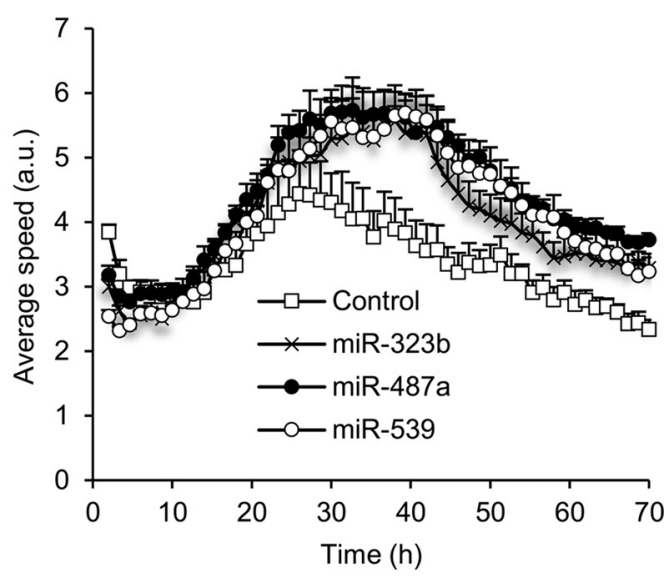

C

Control

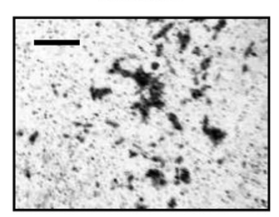

$\mathrm{miR}-323 \mathrm{~b}$

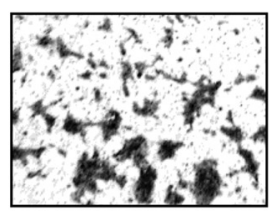

miR-487a

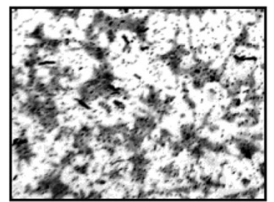

miR-539

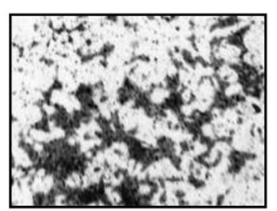

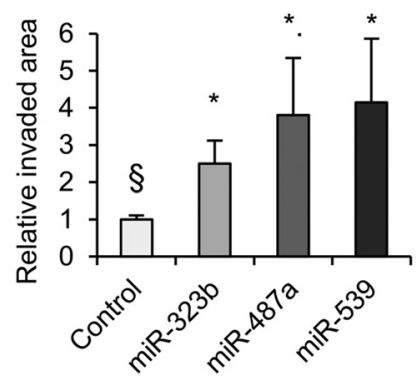

D

OVOL2

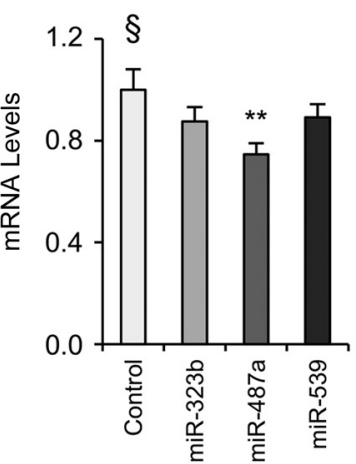

EPHA4

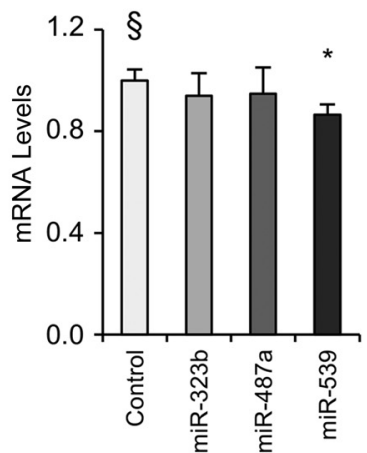

KIF20A

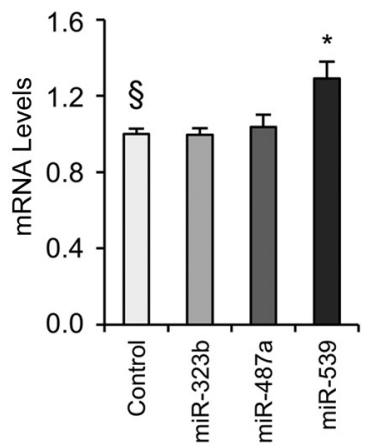

$A X L$

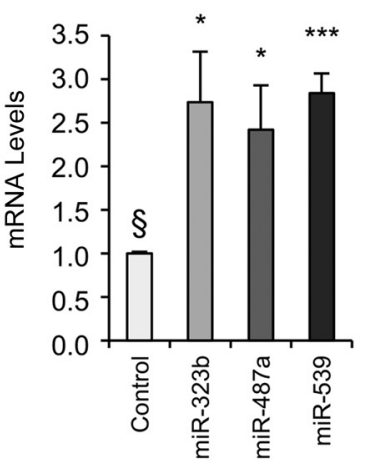

Axl

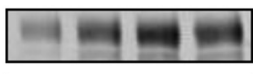

$\beta$-Actin

Figure 6.

Increased levels of miR-323b, miR-487a, or miR-539 induce migration and invasion of lung adenocarcinoma cells. A, Effect of miR-323b, miR-487a, and miR-539 mimic transfection on cell migration of lung adenocarcinoma H2009 cells over a gap of $500 \mu \mathrm{m}$ after 40 hours monitored by time-lapse microscopy. B, Speed dynamics of transfected cells as a function of time (absolute velocities were averaged over the entire frame). Both maximum and overall speeds were increased by the miRNAs. C, Invasion of H2009 cells transfected with the different miRNA mimics through a Matrigel basement membrane matrix after 48 hours. The graph shows quantification of four independent experiments. Scale bar, $500 \mu \mathrm{m}$. D, Modulation of metastasis-related genes by miR-323b, miR-487a, and miR-539. Expression levels were determined by qRT-PCR and analyzed by the $2^{-\Delta \Delta c_{\mathrm{t}}}$ method. Student $t$ test was used for statistical comparison $\left(*, P \leq 0.05 ;{ }^{* *}, P \leq 0.01 ; * * *, P \leq 0.001 ; \S\right.$ control). 
of neoadjuvant and/or adjuvant chemotherapy besides surgical resection (28). Therefore, it is crucial to detect and inhibit tumor cell spread to improve lung cancer patients' outcome.

On the basis of high-dimensional expression data derived from lung adenocarcinoma patients, we identified 28 miRNAs whose expression was significantly associated with lymph node metastasis. The relevance of our approach was confirmed by previous studies showing the dysregulation and functional relevance of some of these candidates in different solid tumors. For example, downregulation of mir-206 and upregulation of mir-134 and mir-370 in lung cancer were associated with increased tumor cell migration, EMT, or decreased patient survival, respectively (29-31). Moreover, in other cancer types such as breast and colorectal cancers, upregulation of mir-409, mir-487a, mir-889, and miR-508 as well as downregulation of mir-598 were related to increased aggressiveness $(10,32-35)$.

Remarkably, more than half of the identified 28 miRNAs in our study belonged to the same genomic cluster on chromosome $14 q 32$. These results are supported by a previous publication showing that 11 miRNAs from this cluster were overexpressed in lung adenocarcinoma patients with worse survival (31). Moreover, the 12qF1 cluster in mice (orthologous to the human 14q32 miRNA cluster) was found to be upregulated in murine lung adenocarcinomas derived from a Kras-mutant mouse model (36). In addition, miRNAs in this cluster have been reported to be regulated during lung development in humans and mice, suggesting that they could be involved in epithelial cell differentiation (37). Finally, activity of the $14 \mathrm{q} 32$ locus was associated with pluripotency of mouse stem cells (38). Thus, several lines of evidence suggest that reactivation of the $14 \mathrm{q} 32$ cluster and its miRNAs support tumor development and progression; however, the functional relevance of coordinated miRNA expression remained elusive.

To study the effects of 14q32 miRNAs on migration and invasion in lung adenocarcinoma cells, we generated stable 14q32 miRNA-overexpressing cell lines using a novel CRISPR technology (CRISPRa), which allowed overexpression of the endogenous miRNAs in a coordinated manner $(17,18)$. To our knowledge, this is the first time that CRISPRa technology has been used for activation of a miRNA cluster. For this, we focused on the region containing the highest density of miRNAs associated with lymph node metastasis (from mir539 to mir-369; Fig. 1C) and validated the successful cluster reactivation by testing miRNAs that are distributed along the $10 \mathrm{~kb}$ cluster section downstream of the sgRNA-binding sites. Although additional elements of the cluster besides miRNAs might also be involved in tumor progression, as it has been reported before for noncoding RNA MEG3 (39), we confirmed that none of the genes/lncRNAs adjacent to the miRNA cluster were overexpressed in CRISPRa-14q32 cells. This approach allowed us to demonstrate that the coordinated overexpression of $14 \mathrm{q} 32$ miRNAs induced tumor migration and invasion in lung adenocarcinoma tumor cells, without affecting cell viability. Importantly, increased cellular levels of the three selected miRNAs miR-323b, miR-487a, and miR-539 were sufficient to induce significant migration and invasion in lung adenocarcinoma cells. These findings support the hypothesis that $14 \mathrm{q} 32$ miRNAs cooperate in regulating tumor cell dissemination. However, whether and to what extent other $14 \mathrm{q} 32$ cluster miRNAs participate in this cellular response (e.g., miR-889 and
miR-134) needs to be tested. The observed delayed effect on lung cancer cell migration after miRNA mimic transfection ( $>20$ hours) may be due to the time needed for the initiation of molecular and cellular processes (40). Furthermore, our data indicate that indirect mechanisms regulating migrationrelevant proteins might also be responsible for the observed delay in promoting cell motility.

As the overexpressed $14 \mathrm{q} 32$ miRNAs were coregulated in lung adenocarcinoma tissue samples from both discovery and validation patient cohorts, we hypothesized that they share a common regulatory upstream mechanism. Because amplification of the $14 \mathrm{q} 32$ locus was previously reported to be rare in lung adenocarcinoma patients $(<1 \%$; ref. 31$)$, we hypothesized that disruption of epigenetic mechanisms such as DNA methylation, a typical hallmark of cancer (11), may facilitate coordinated overexpression of the $14 \mathrm{q} 32$ miRNAs. In this regard, the effect of such epigenetic aberrancies on miRNA expression is well documented in human tumors, where many miRNAs have already been found up- and downregulated due to cancerrelated DNA methylation changes (41). Importantly, our methylation analyses did not support the possibility that a LOI of the DLK1-DIO3 locus could play a role in metastasis and tumor cell dissemination of lung adenocarcinoma. However, the analyses illustrated a gradual decrease in DNA methylation of the 14q32 miRNA cluster from healthy lung tissue to N0 stage tumors and tumors with lymph node metastasis. This gradual hypomethylation correlated with the overexpression of the $14 \mathrm{q} 32$ miRNAs. As mentioned before, different reports have shown how aberrant DNA hypomethylation can lead to miRNA overexpression in lung and other cancers (42), but a recent article revealed that the DLK1-DIO3 locus, which comprises the $14 \mathrm{q} 32$ miRNA cluster, is also hypomethylated in smokingassociated non-small cell lung cancer compared with normal lung tissue.

Notably, miRNAs are considered as potential diagnostic and prognostic cancer biomarkers as they are detectable in tissues and several biofluids, such as blood, urine, or sputum (4). Although prognostic markers are currently not implemented in clinical routine work of lung adenocarcinoma, this information could be relevant for aftercare of patients after resection or adjuvant therapy and might be of importance for future targeted therapy. Especially, the accessibility of miRNAs in biofluids might represent novel possibilities for future prognostic marker panels not only for clinical decision making but also for appropriate stratification of future clinical trials. As miR-323b, miR-487a, and miR-539 were significantly upregulated in lung adenocarcinoma patients with lymph node metastasis and involved in tumor cell motility, we analyzed their possible role in predicting patient DFS. Interestingly, overexpression of the three miRNAs was associated with a shorter time to relapse in never-smoker patients, whereas this association was not found in smoker patients Remarkably, although the levels of miR-323b, miR-487a, and miR-539 were associated with lymph node metastasis, association of $\mathrm{N}$ stage with DFS in never-smokers did not reach the level of significance, illustrating that expression of these miRNAs may be a better prognostic marker than $\mathrm{N}$ stage in this patient group. Indeed, correction by confounding clinical variables ( $\mathrm{N}$ stage, $\mathrm{T}$ stage, and cancer stage) showed that miRNA expression might represent an independent prognostic biomarker in this patient subgroup. Although one limitation of this study is the relative low 
number of events in the never-smoker group, the different prognostic value of the miRNAs in smoker and nonsmoker patients could be explained by the relative importance of miRNA overexpression compared with the plethora of mechanisms to induce cell migration that have been attributed to smoking, such as increasing promigratory cytokines as CCL20 and activation of PAK6, a kinase associated with the metastatic process $(43,44)$. The possible differential effect of the $14 q 32$ miRNA cluster in disrupting different signaling pathways in lung cancer patients according to their smoking history warrants further investigation.

Our results indicate that multiple genes involved in motility-related processes (OVOL2, EPHA4, AXL, LOXL2, CDH2, KIF3C, and KIF20A) have a role in the $14 \mathrm{q} 32$ miRNA-mediated tumor cell dissemination in lung adenocarcinoma. miR323b, miR-487a, and miR-539, which individually induce lung adenocarcinoma cell migration and invasion, significantly contribute to the modulation of several of the aforementioned migration-related genes: miR-539 induced the upregulation of KIF20A and $A X L$ and the downregulation of EPHA4, while miR-323b increased $A X L$, and miR-487a decreased OVOL2 and increased AXL. However, our studies, which included luciferase assays, indicated that they are not direct targets of the miRNAs (data not shown). With the exception of $A X L$, which is regulated by all three tested miRNAs, moderate effects of individual miR-323b, miR$487 \mathrm{a}$, and miR-539 on these target genes were detectable. This further supports the idea that miRNAs on the one hand facilitate their biological functions through a plethora of downstream target genes (45) and that on the other hand the cooperative activation of clustered $14 \mathrm{q} 32$ miRNAs is necessary to facilitate a pronounced migratory response in this experimental setup.

\section{Disclosure of Potential Conflicts of Interest}

P. Schirmacher has received speakers bureau honoraria from AstraZeneca and is a consultant/advisory board member for AstraZeneca, BMS, Novartis,

\section{References}

1. Wood SL, Pernemalm M, Crosbie PA, Whetton AD. The role of the tumormicroenvironment in lung cancer-metastasis and its relationship to potential therapeutic targets. Cancer Treat Rev 2014;40:558-66.

2. Du L, Pertsemlidis A. MicroRNAs and lung cancer: tumors and 22-mers. Cancer Metastasis Rev 2010;29:109-22.

3. Vannini I, Fanini F, Fabbri M. MicroRNAs as lung cancer biomarkers and key players in lung carcinogenesis. Clin Biochem 2013;46:918-25.

4. MacDonagh L, Gray SG, Finn SP, Cuffe S, O'Byrne KJ, Barr MP. The emerging role of microRNAs in resistance to lung cancer treatments. Cancer Treat Rev 2015;41:160-9.

5. Zhang WC, Liu J, Xu X, Wang G. The role of microRNAs in lung cancer progression. Med Oncol 2013;30:675.

6. Bender E. Epidemiology: the dominant malignancy. Nature 2014; 513:S2-3.

7. Geyh S, Rodriguez-Paredes M, Jager P, Khandanpour C, Cadeddu RP, Gutekunst J, et al. Functional inhibition of mesenchymal stromal cells in acute myeloid leukemia. Leukemia 2016;30:683-91.

8. Aryee MJ, Jaffe AE, Corrada-Bravo H, Ladd-Acosta C, Feinberg AP, Hansen KD, et al. Minfi: a flexible and comprehensive Bioconductor package for the analysis of Infinium DNA methylation microarrays. Bioinformatics 2014; 30:1363-9.

9. Chang RM, Xiao S, Lei X, Yang H, Fang F, Yang LY. miRNA-487a promotes proliferation and metastasis in hepatocellular carcinoma. Clin Cancer Res 2017;23:2593-604. and Roche. No potential conflicts of interest were disclosed by the other authors.

\section{Authors' Contributions}

Conception and design: M. González-Vallinas, K. Breuhahn

Development of methodology: M. González-Vallinas, M. Rodríguez-Paredes, F.J. Sánchez-Rivera, N.S. Müller, D.F. Tschaharganeh

Acquisition of data (provided animals, acquired and managed patients, provided facilities, etc.): M. González-Vallinas, J. Schmitt, C. De La Torre, A. Warth, N. Gretz, T. Muley, M. Meister

Analysis and interpretation of data (e.g., statistical analysis, biostatistics, computational analysis): M. González-Vallinas, M. RodríguezParedes, M. Albrecht, C. Sticht, D. Stichel, J. Gutekunst, A. Pitea, S. Sass, J. Lorenzo-Bermejo, J. Schmitt, C. De La Torre, F.J. Theis, N.S. Müller, N. Gretz, F. Matthäus, K. Breuhahn

Writing, review, and/or revision of the manuscript: M. González-Vallinas, M. Rodríguez-Paredes, S. Sass, J. Lorenzo-Bermejo, A. Warth, N. Gretz, T. Muley, M. Meister, P. Schirmacher, K. Breuhahn

Administrative, technical, or material support (i.e., reporting or organizing data, constructing databases): T. Muley, M. Meister, P. Schirmacher, K. Breuhahn Study supervision: F.J. Theis, K. Breuhahn

\section{Acknowledgments}

This work was supported in part by the BMBF (LungSysII, grant number FKZ 0316042B to K. Breuhahn) and the University of Heidelberg (Stiftung für Krebs- und Scharlachforschung to M. González-Vallinas). M. GonzálezVallinas was supported by the Alfonso Martin Escudero Foundation. We also thank the TCGA Research Network and the research groups involved in the lung adenocarcinoma project for making data public. Tissue samples were provided by the tissue bank of the National Center for Tumor Diseases (NCT; Heidelberg, Germany) in accordance with the regulations of the tissue bank and the approval of the Ethics Committee of Heidelberg University. We are especially grateful for the contribution of lung adenocarcinoma specimen donors.

The costs of publication of this article were defrayed in part by the payment of page charges. This article must therefore be hereby marked advertisement in accordance with 18 U.S.C. Section 1734 solely to indicate this fact.

Received June 27, 2017; revised September 18, 2017; accepted December 8, 2017; published OnlineFirst January 12, 2018.

10. Ma M, He M, Jiang Q, Yan Y, Guan S, Zhang J, et al. MiR-487a promotes TGF-beta1-induced EMT, the migration and invasion of breast cancer cells by directly targeting MAGI2. Int J Biol Sci 2016;12:397-408.

11. Rodriguez-Paredes M, Esteller M. Cancer epigenetics reaches mainstream oncology. Nat Med 2011;17:330-9.

12. Berman BP, Weisenberger DJ, Aman JF, Hinoue T, Ramjan Z, Liu Y, et al. Regions of focal DNA hypermethylation and long-range hypomethylation in colorectal cancer coincide with nuclear lamina-associated domains. Nat Genet 2011;44:40-6.

13. Kircher M, Bock C, Paulsen M. Structural conservation versus functional divergence of maternally expressed microRNAs in the Dlk1/Gtl2 imprinting region. BMC Genomics 2008;9:346

14. Benetatos L, Hatzimichael E, Londin E, Vartholomatos G, Loher P, Rigoutsos I, et al. The microRNAs within the DLK1-DIO3 genomic region: involvement in disease pathogenesis. Cell Mol Life Sci 2013;70: 795-814.

15. Kagami M, O'Sullivan MJ, Green AJ, Watabe Y, Arisaka O, Masawa N, et al. The IG-DMR and the MEG3-DMR at human chromosome 14q32.2 hierarchical interaction and distinct functional properties as imprinting control centers. PLoS Genet 2010;6:e1000992.

16. Manodoro F, Marzec J, Chaplin T, Miraki-Moud F, Moravcsik E, Jovanovic $\mathrm{JV}$, et al. Loss of imprinting at the $14 \mathrm{q} 32$ domain is associated with microRNA overexpression in acute promyelocytic leukemia. Blood 2014; 123:2066-74. 
17. Chavez A, Scheiman J, Vora S, Pruitt BW, Tuttle M, PR Iver E, et al. Highly efficient Cas9-mediated transcriptional programming. Nat Methods 2015; 12:326-8.

18. Kiani S, Chavez A, Tuttle M, Hall RN, Chari R, Ter-Ovanesyan D, et al. Cas9 gRNA engineering for genome editing, activation and repression. Nat Methods 2015;12:1051-4.

19. Saintigny P, Peng S, Zhang L, Sen B, Wistuba II, Lippman SM, et al. Global evaluation of Eph receptors and ephrins in lung adenocarcinomas identifies EphA4 as an inhibitor of cell migration and invasion. Mol CancerTher 2012;11:2021-32.

20. Wang ZH, Li Z, Hu M, Yang QJ, Yan S, Wu RS, et al. Ovol2 gene inhibits the Epithelial-to-Mesenchymal Transition in lung adenocarcinoma by transcriptionally repressing Twist1. Gene 2017;600:1-8.

21. Grinberg-Rashi H, Ofek E, Perelman M, Skarda J, Yaron P, Hajduch M, et al. The expression of three genes in primary non-small cell lung cancer is associated with metastatic spread to the brain. Clin Cancer Res 2009;15: 1755-61.

22. Ishikawa M, Sonobe M, Nakayama E, Kobayashi M, Kikuchi R, Kitamura J, et al. Higher expression of receptor tyrosine kinase Axl, and differential expression of its ligand, Gas6, predict poor survival in lung adenocarcinoma patients. Ann Surg Oncol 2013;20:S467-76.

23. Zhan P, Lv XJ, Ji YN, Xie H, Yu LK. Increased lysyl oxidase-like 2 associates with a poor prognosis in non-small cell lung cancer. Clin Respir J 2016 Nov 18. [Epub ahead of print].

24. Wang C, Wang C, Wei Z, Li Y, Wang W, Li X, et al. Suppression of motor protein KIF3C expression inhibits tumor growth and metastasis in breast cancer by inhibiting TGF-beta signaling. Cancer Lett 2015;368:105-14

25. Zhang W, He W, Shi Y, Gu H, Li M, Liu Z, et al. High expression of KIF20A is associated with poor overall survival and tumor progression in early-stage cervical squamous cell carcinoma. PLoS One 2016;11:e0167449.

26. Muller B, Bovet M, Yin Y, Stichel D, Malz M, Gonzalez-Vallinas M, et al. Concomitant expression of far upstream element (FUSE) binding protein (FBP) interacting repressor (FIR) and its splice variants induce migration and invasion of non-small cell lung cancer (NSCLC) cells. J Pathol 2015;237:390-401.

27. Chan SW, Lim CJ, Guo K, Ng CP, Lee I, Hunziker W, et al. A role for TAZ in migration, invasion, and tumorigenesis of breast cancer cells. Cancer Res 2008;68:2592-8.

28. Yeh YC, Kadota K, Nitadori J, Sima CS, Rizk NP, Jones DR, et al. International Association for the Study of Lung Cancer/American Thoracic Society/ European Respiratory Society classification predicts occult lymph node metastasis in clinically mediastinal node-negative lung adenocarcinoma. Eur J Cardiothorac Surg 2016;49:e9-e15.

29. Chen X, Tong ZK, Zhou JY, Yao YK, Zhang SM, Zhou JY. MicroRNA-206 inhibits the viability and migration of human lung adenocarcinoma cells partly by targeting MET. Oncol Lett 2016;12:1171-7.

30. Kitamura K, Seike M, Okano T, Matsuda K, Miyanaga A, Mizutani H, et al. MiR-134/487b/655 cluster regulates TGF-beta-induced epithelial-mesenchymal transition and drug resistance to gefitinib by target- ing MAGI2 in lung adenocarcinoma cells. Mol Cancer Ther 2014; 13:444-53.

31. Nadal E, Zhong J, Lin J, Reddy RM, Ramnath N, Orringer MB, et al. A MicroRNA cluster at $14 \mathrm{q} 32$ drives aggressive lung adenocarcinoma. Clin Cancer Res 2014;20:3107-17.

32. Josson S, Gururajan M, Sung SY, Hu P, Shao C, Zhau HE, et al. Stromal fibroblast-derived miR-409 promotes epithelial-to-mesenchymal transition and prostate tumorigenesis. Oncogene 2015;34:2690-9.

33. Lin $C$, Liu A, Zhu J, Zhang $X, W u ~ G$, Ren $P$, et al. miR-508 sustains phosphoinositide signalling and promotes aggressive phenotype of oesophageal squamous cell carcinoma. Nat Commun 2014;5:4620.

34. Molina-Pinelo S, Carnero A, Rivera F, Estevez-Garcia P, Bozada JM, Limon $M L$, et al. MiR-107 and miR-99a-3p predict chemotherapy response in patients with advanced colorectal cancer. BMC Cancer 2014;14:656.

35. Zhao BS, Liu SG, Wang TY, Ji YH, Qi B, Tao YP, et al. Screening of microRNA in patients with esophageal cancer at same tumor node metastasis stage with different prognoses. Asian Pac J Cancer Prev 2013;14:139-43.

36. Valdmanis PN, Roy-Chaudhuri B, Kim HK, Sayles LC, Zheng Y, Chuang $\mathrm{CH}$, et al. Upregulation of the microRNA cluster at the Dlk1-Dio3 locus in lung adenocarcinoma. Oncogene 2015;34:94-103.

37. Williams AE, Moschos SA, Perry MM, Barnes PJ, Lindsay MA. Maternally imprinted microRNAs are differentially expressed during mouse and human lung development. Dev Dyn 2007;236:572-80.

38. Liu L, Luo GZ, Yang W, Zhao X, Zheng Q, Lv Z, et al. Activation of the imprinted Dlk1-Dio3 region correlates with pluripotency levels of mouse stem cells. J Biol Chem 2010;285:19483-90.

39. Liu J, Wan L, Lu K, Sun M, Pan X, Zhang P, et al. The long noncoding RNA MEG3 contributes to cisplatin resistance of human lung adenocarcinoma. PLoS One 2015;10:e0114586.

40. Eichhorn SW, Guo H, McGeary SE, Rodriguez-Mias RA, Shin C, Baek D et al. mRNA destabilization is the dominant effect of mammalian microRNAs by the time substantial repression ensues. Mol Cell 2014;56:104-15.

41. Suzuki H, Maruyama R, Yamamoto E, Kai M. DNA methylation and microRNA dysregulation in cancer. Mol Oncol 2012;6:567-78.

42. Iorio MV, Visone R, Di Leva G, Donati V, Petrocca F, Casalini P, et al. MicroRNA signatures in human ovarian cancer. Cancer Res 2007;67: 8699-707

43. Raja R, Sahasrabuddhe NA, Radhakrishnan A, Syed N, Solanki HS, Puttamallesh VN, et al. Chronic exposure to cigarette smoke leads to activation of p21 (RAC1)-activated kinase 6 (PAK6) in non-small cell lung cancer cells. Oncotarget 2016;7:61229-45.

44. Wang GZ, Cheng X, Li XC, Liu YQ, Wang XQ, Shi X, et al. Tobacco smoke induces production of chemokine CCL20 to promote lung cancer. Cancer Lett 2015;363:60-70.

45. Cherni I, Weiss GJ. miRNAs in lung cancer: large roles for small players. Future Oncol 2011;7:1045-55.

46. Altman DG, Lausen B, Sauerbrei W, Schumacher M. Dangers of using "optimal" cutpoints in the evaluation of prognostic factors. J Natl Cancer Inst 1994;86:829-35. 


\section{Molecular Cancer Research}

\section{Epigenetically Regulated Chromosome 14q32 miRNA Cluster Induces Metastasis and Predicts Poor Prognosis in Lung Adenocarcinoma Patients}

Margarita González-Vallinas, Manuel Rodríguez-Paredes, Marco Albrecht, et al.

Mol Cancer Res 2018;16:390-402. Published OnlineFirst January 12, 2018.

Updated version Access the most recent version of this article at: doi:10.1158/1541-7786.MCR-17-0334

Supplementary Access the most recent supplemental material at: Material http://mcr.aacrjournals.org/content/suppl/2018/01/12/1541-7786.MCR-17-0334.DC1

Cited articles This article cites 45 articles, 9 of which you can access for free at: http://mcr.aacrjournals.org/content/16/3/390.full\#ref-list-1

Citing articles This article has been cited by 3 HighWire-hosted articles. Access the articles at: http://mcr.aacrjournals.org/content/16/3/390.full\#related-urls

E-mail alerts Sign up to receive free email-alerts related to this article or journal.

Reprints and To order reprints of this article or to subscribe to the journal, contact the AACR Publications Department at Subscriptions pubs@aacr.org.

Permissions To request permission to re-use all or part of this article, use this link http://mcr.aacrjournals.org/content/16/3/390.

Click on "Request Permissions" which will take you to the Copyright Clearance Center's (CCC) Rightslink site. 